\section{If your name's not down, you're not coming in}

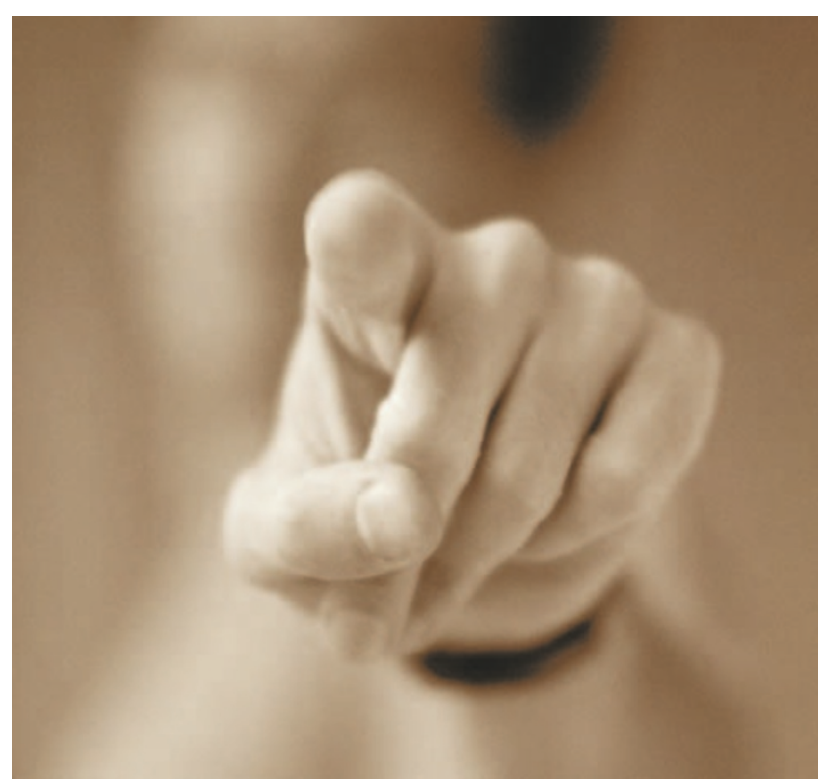

According to a recent report in Science, CD $8 \alpha \alpha$ could be the longsought marker of effector $\mathrm{T}$ cells that are destined to enter the memory pool. Only a small fraction of the effector T-cell population that expands during a primary response survives as long-lived memory $\mathrm{T}$ cells after antigen has been cleared, but until now, the mechanism of this selection process has been unclear.

Madakamutil et al. used tetramers of the non-classical MHC class I molecule thymic leukaemia antigen (TL) - which is a unique ligand for CD $8 \alpha \alpha$ - to show that mature $\mathrm{CD} 8 \alpha \beta^{+} \mathrm{T}$ cells upregulate the cellsurface expression of CD $8 \alpha \alpha$ after stimulation through CD3. CD8 $\alpha \alpha$ upregulation was also seen after stimulation of OT-1 T-cell receptor (TCR)-transgenic splenocytes with their cognate antigen (ovalbumin, OVA). This effect was greatest when OT-1 splenocytes were stimulated with OVA presented by TL-expressing antigen-presenting cells, indicating that interaction between $\mathrm{CD} 8 \alpha \alpha$ and
TL is necessary to maintain CD8 $\alpha \alpha$ expression. TL is expressed by activated dendritic cells and monocytes, indicating that such an interaction could occur physiologically.

Expression of CD8 $\alpha \alpha$ correlated with high levels of expression of the anti-apoptotic proteins Bcl-2 and $\mathrm{Bcl}-\mathrm{X}_{\mathrm{L}}$ and with increased T-cell survival in vitro. These results were confirmed in vivo after infection of mice with lymphocytic choriomeningitis virus (LCMV); CD8 $\alpha \alpha$ was upregulated on a subset of LCMV-specific CD $8 \alpha \beta^{+}$T cells after infection, and these $\mathrm{T}$ cells expressed high levels of Bcl- $\mathrm{X}_{\mathrm{L}}$ and survived long term (for up to 60 days after infection).

As CD $8 \alpha \alpha$ is only upregulated on a subset of effector T cells and promotes long-term survival, it might be a marker of memory T-cell precursors. To test this, they transferred TCR-transgenic P14 T cells (specific for an LCMV peptide) to naive recipients, then re-isolated the P14 T cells 8 days after LCMV

\title{
LYMPHOCYTE SIGNALLING
}

\section{Lay your bets: $2-1$ on NK and NKT cells}

The lymphoid-restricted T-box transcription factor T-bet is known to have a key role in the generation of $\mathrm{T}$ helper 1-cell immune responses. Now, an in depth analysis of T-betdeficient mice, published in Immunity, indicates that this transcription factor also regulates the late stages of development of natural killer (NK) cells and of NKT cells that express the semi-invariant $\mathrm{V} \alpha 14 \mathrm{~T}$-cell receptor (TCR) - V $\alpha 14 i$ NKT cells.

C57BL/6 mice express the NK-cell receptors NK1.1 and Ly49d. Therefore, to assess the role of T-bet in NK-cell development and function, T-bet-deficient mice were backcrossed onto the C57BL/6 background. Flow cytometric analysis indicated that T-bet-deficient mice had reduced numbers of NK cells in the spleen, liver and peripheral blood, and that the remaining cells had an immature phenotype - as determined by reduced expression of $\mathrm{CD} 43, \mathrm{CD} 11 \mathrm{~b}$ and DX5, and increased expression of c-Kit but expressed a normal repertoire of activating and inhibitory NK-cell receptors. Similarly, using $\alpha$-galactosyl ceramideloaded CD1d tetramers to identify $\mathrm{V} \alpha 14 i$ NKT cells, T-bet-deficient mice were shown to have almost no $\mathrm{V} \alpha 14 i \mathrm{NKT}$ cells in the liver, spleen and thymus. By contrast, $\mathrm{V} \alpha 14 i$ NKT-cell maturation intermediates in the thymus, previously defined as tetramerpositive cells expressing CD44 but not NK1.1 or Ly49 receptors, were detected in T-betdeficient mice, indicating that, as for NK cells, V $\alpha 14 i$ NKT-cell terminal maturation is blocked in the absence of T-bet.

By using wild-type or T-bet-deficient bone marrow to reconstitute wild-type and T-betdeficient mice, the authors showed that the defect in NK-cell and V $\alpha 14 i$ NKT-cell development was intrinsic to T-bet-deficient bone marrow. Furthermore, when a mixture of wild-type and T-bet-deficient bone marrow was used to reconstitute a T-bet-sufficient host, the NK cells and V $\alpha 14 i$ NKT cells were largely derived from the wild-type bone marrow, indicating a stem-cell-intrinsic role for T-bet in the development of the NK-cell and V $\alpha 14 i$ NKT-cell lymphoid compartments. Consistent with a cell-intrinsic role in terminal maturation, overexpression of T-bet in T-bet-deficient haematopoietic stem cells increased the number and maturation status of NK cells in the spleen.
To further determine the role of T-bet in NK-cell and $V \alpha 14 i$ NKT-cell maturation, the authors analysed the effector functions of T-bet-deficient cells. Interleukin-12 (IL-12) and IL-18 stimulation of NK cells results in a rapid burst of interferon- $\gamma$ $($ IFN- $\gamma$ ) secretion that was not impaired in T-bet-deficient NK cells. However, T-bet was required to maintain IFN- $\gamma$ production during prolonged NK-cell stimulation. By contrast, T-bet-deficient V $\alpha 14 i$ NKT cells were unable to produce IFN- $\gamma$ when stimulated in vivo with $\alpha$-galactosyl ceramide. The authors suggest that this distinct dependence on T-bet for regulating IFN- $\gamma$ production could result from redundancy between T-box transcription factors, as NK cells but not V $\alpha 14 i$ NKT cells express the T-box-family member eomesodermin.

This study identifies a novel role for T-bet as a regulator of the terminal differentiation of NK cells and V $\alpha 14 i$ NKT cells. However, further studies are required to determine whether the molecular role of this transcription factor is the same in these two cell types.

Karen Honey

(2) References and links ORIGINAL RESEARCH PAPER Townsend, M. J. et al. T-bet regulates the terminal maturation and homeostasis of NK and Vo14i NKT cells. Immunity 20, 477-494 (2004) 
infection. CD8 $\alpha \alpha^{+}$and CD8 $\alpha \alpha^{-}$ P14 fractions were then transferred separately to naive recipients, and the mice were challenged with LCMV 40 days later. Only those mice that had received the $\mathrm{CD} 8 \alpha \alpha^{+}$ fraction had a significant memory P14 T-cell response to the virus, and their $\mathrm{T}$ cells produced interferon- $\gamma$ in vitro when stimulated with LCMV peptide. An enhancer deletion in the mouse $C d 8$ locus that prevents the high-level expression of CD $8 \alpha$ required to form CD $8 \alpha \alpha$ homodimers, but still allows $\mathrm{CD} 8 \alpha \beta$ heterodimer formation, did not affect the primary response to LCMV but led to low numbers and a poor in vitro response of LCMV-specific $\mathrm{T}$ cells 50 days after infection, which confirms the essential role of $\mathrm{CD} 8 \alpha \alpha$ in T-cell survival and memory-cell differentiation.

Kirsty Minton

(2) References and links ORIGINAL RESEARCH PAPER Madakamutil, L. T. et al. CD8 $\alpha \alpha$-mediated survival and differentiation of $C D 8$ memory T-cell precursors. Science 304, 590-593 (2004)

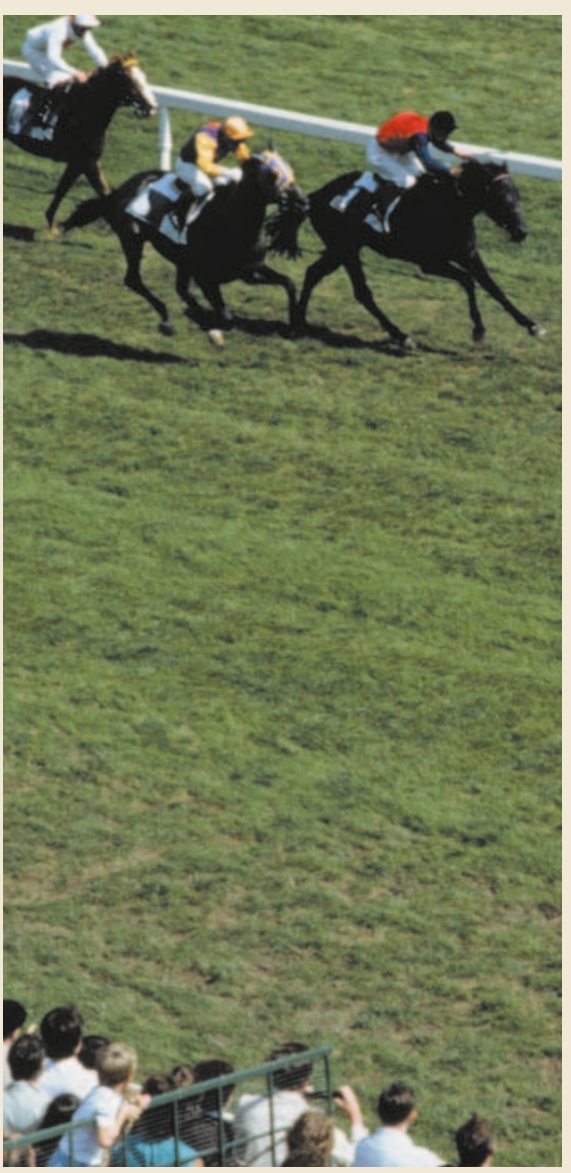

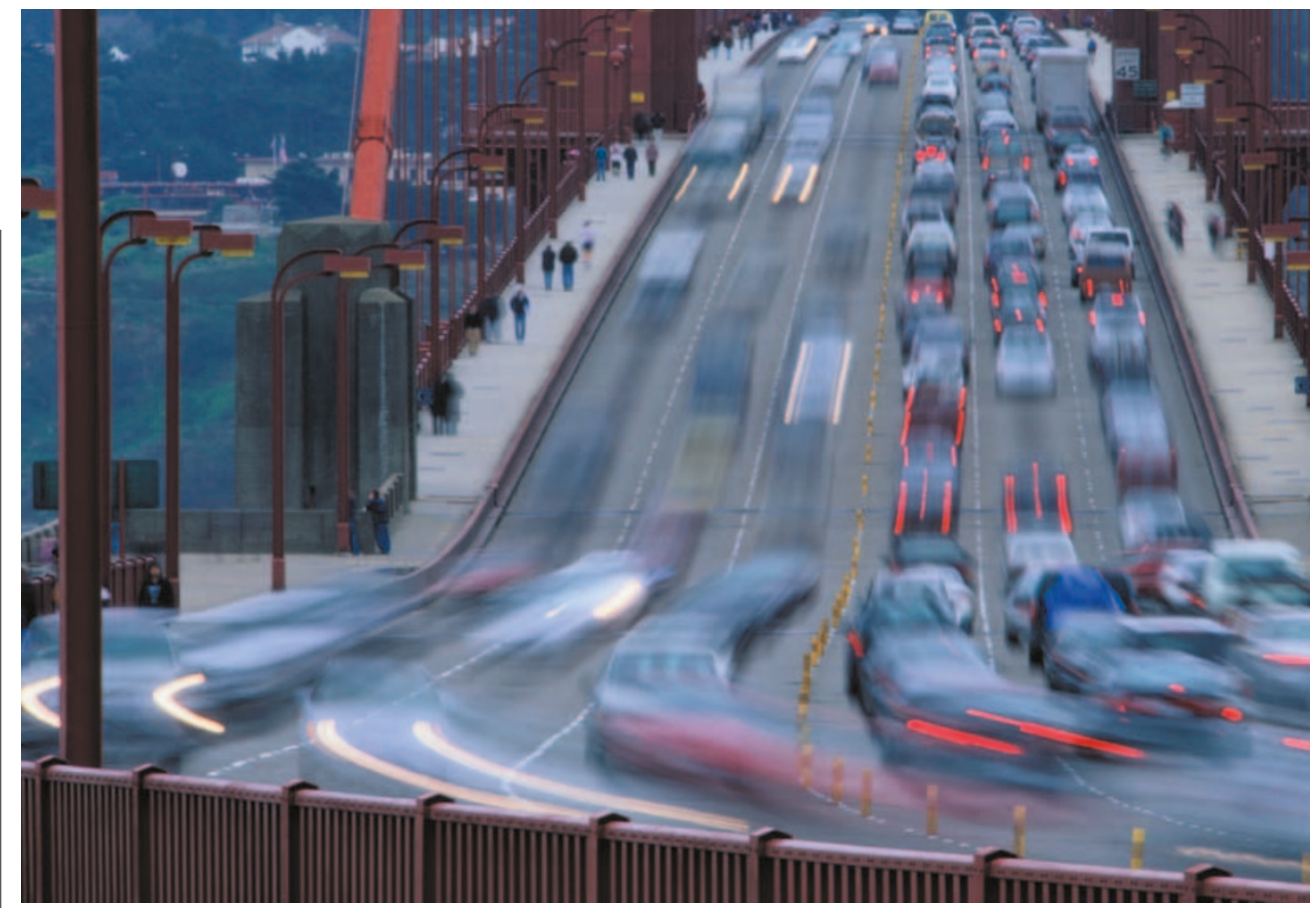

\section{INNATE IMMUNITY}

\section{Destroying Tolls}

Toll-like receptor (TLR) signalling generates a pro-inflammatory immune response that is capable not only of clearing infection but also of damaging host tissues. It is therefore important to tightly regulate the TLR-induced response, and a paper published recently in Nature Immunology describes a novel mechanism for doing just that — TLR ubiquitylation and targeting for degradation.

Ubiquitin targeting of proteins for proteolytic degradation is a three-step process. First, ubiquitin is activated by a ubiquitin-activating enzyme (E1); second, activated ubiquitin is transferred to a ubiquitin-conjugating enzyme (E2 or UBC); and third, an E3 ubiquitin ligase attaches ubiquitin to lysine residues on the protein to be targeted for degradation.

Chuang and Ulevitch reasoned that the cytoplasmic Toll/interleukin-1-receptor (TIR) domain of TLRs, which is crucial for recruiting proteins involved in TLR signalling, might also bind proteins involved in negatively regulating TLRs. Using a yeast two-hybrid approach, they found that the TIR domain of human TLR9 binds a previously described protein known as TRIAD3. This interaction was not specific for the TIR domain of TLR9, as TRIAD3 also interacted with TLR3, TLR4 and TLR5, but it did not interact with TLR2.

Further analysis of TRIAD3 identified five splice variants, designated TRIAD3A-E, of which TRIAD3A was the most ubiquitous and abundant. TRIAD3A was predicted to contain RING motifs characteristic of the RING-finger group of E3 ligases. Consistent with this, TRIAD3A was shown to interact with some
E2 enzymes - UBCH7 and to some extent $\mathrm{UBCH} 8$, but not with $\mathrm{UBCH} 2$ or $\mathrm{UBCH} 10$. Furthermore, when overexpressed in the 293 cell line, TRIAD3A induced auto-ubiquitylation, as well as ubiquitylation of TLR9 but not TLR2.

In separate studies, TRIAD3A promoted degradation of TLR9 and TLR4, but had no effect on the level of TLR2. TRIAD3A was shown to function in a dose-dependent manner, and degradation of TLRs was abrogated in the presence of a proteasome inhibitor but not by inhibitors of lysosomal proteolysis. The decrease in TLR protein levels was concomitant with reduced signalling in response to stimulation with TLR4 and TLR9 ligands, whereas TLR2 ligands induced a normal signalling response.

Together, these data indicate that TRIAD3A is an E3 ligase that regulates the ubiquitylation of specific TLRs, targeting them for proteasomal degradation. Further evidence for this was provided by the observation that TRIAD3Aspecific small interfering RNAs (siRNAs), which substantially reduce the expression of TRIAD3A protein, increased the basal level of TLR9 expression, and abolished the decrease in TLR9 expression that is normally observed after stimulation with TLR9 ligands. Similarly, TRIAD3A-specific siRNAs induced increased activation after exposure to TLR4 ligands but not TLR2 ligands.

This study identifies a novel mechanism for negative regulation of specific TLR-signalling pathways - TRIAD3A-regulated ubiquitindependent proteasome degradation. Future studies will no doubt focus on how TRIAD3A is recruited to the TLR-signalling complex to mediate its specific regulatory effects.

(2) References and links

Karen Honey ORIGINAL RESEARCH PAPER Chuang, T. \& Ulevitch, R. J. Triad3A an E3 ubiquitin-protein ligase regulating Toll-like receptors. Nature Immunol. 25 April 2004 (doi:10.1038/ni1066) 